\title{
Sistemas de produção de base ecológica: Uma alternativa para o desenvolvimento
}

\section{sustentável}

\author{
Ecologically based production systems: An alternative for sustainable development \\ Sistemas de producción de base ecológica: Uma alternativa para el desarrolho sostenible
}

Recebido: 25/06/2021 | Revisado: 04/07/2021 | Aceito: 07/07/2021 | Publicado: 17/07/2021

Érika Aparecida de Araújo Soares
ORCID: https://orcid.org/0000-0001-5723-5255
Instituto Federal de Educação, Ciência e Tecnologia do Rio Grande do Norte, Brasil
E-mail: erika.soares01@ hotmail.com
Saint Clair Lira Santos
ORCID: https://orcid.org/0000-0002-2738-2972
E-mail: saint.lira@ @ifrn.edu.br
Laís Kisly Costa Silva
Instituto Federal de Educação, Ciência e Tecnologia do Rio Grande do
ORCID: https://orcid.org/0000-0002-6489-6075
Universidade Federal da Paraíba, Brasil
E-mail: laiskisly@gmail.com
José Eduardo do Nascimento Cardoso
ORCID: https://orcid.org/0000-0003-3955-5532
Faculdade Maurício de Nassau, Brasil
E-mail: eduardocardosonutri@ @hotmail.com
Zandra Lorenna Coutinho de Melo Costa
ORCID: https://orcid.org/0000-0002-9799-4093
E-mail: zandracoutinho@gmail.com

\section{Resumo}

Quando nos referimos à Agroecologia, estamos focalizando um conjunto de princípios (unidade) e, quando tratamos de Agriculturas Ecológicas, nos remetemos às manifestações concretas ou à materialização daqueles conceitos (diversidade), mediante formas de manejo específicas. Esse conjunto de iniciativas/experiências, a partir de uma visão holística, que considera a complexidade e a relação entre os diversos processos que ocorrem nos agroecossitemas, configura um modelo de agricultura com base em princípios ecológicos de produção, que contrapõe à agricultura moderna. O presente artigo teve como objetivo realizar um levantamento bibliográfico acerca dos principais sistemas de produção de base ecológica, com ênfase no sistema orgânico. Através de descritores previamente selecionados realizou-se uma busca ativa nas principais plataformas de pesquisa, por artigos, dissertações e teses que abordassem como tema principal ou secundário as principais formas de agricultura ecológica: Biodinâmica, Natural, Biológica e Orgânica. Muito além da ausência do uso de agrotóxicos, a adesão a uma agricultura de base ecológica, de forma mais específica, a agricultura de base orgânica, é expansiva e envolve questões sociais, ambientais e econômicas. Além de intrinsicamente defender o direito a vida e a saúde, de produtores e consumidores, a agricultura ecológica engaja a prática de uma economia solidária, valorizando os pequenos agricultores e contribuindo para sua independência, além de trazer consigo pontos importantes como sustentabilidade, preservação ambiental, respeito aos saberes locais e não desperdício de alimentos e recursos.

Palavras-chave: Agricultura biodinâmica; Agricultura biológica; Agricultura natural; Agricultura orgânica.

\begin{abstract}
When we refer to Agroecology, we are focusing on a set of principles (unit) and, when we refer to Ecological Agriculture, we refer to concrete manifestations or the materialization of these concepts (diversity), through specific forms of management. This set of initiatives / experiences, in a holistic view, which considers the complexity and the relationship between the various processes that occur in agroecosystems, configures an agricultural model based on ecological principles of production, which is opposed to modern agriculture. This article aimed to carry out a bibliographical survey on the main ecologically-based production systems, with an emphasis on the organic system. Using previously selected descriptors, an active search was carried out in the main research platforms, for articles, dissertations and theses that addressed as main or secondary theme the main forms of ecological agriculture: Biodynamic, Natural, Biological and Organic. Far beyond the absence of the use of pesticides, adherence to ecologically-based agriculture, more specifically, to organic-based agriculture, is expansive and involves social, environmental and economic issues. In addition to intrinsically defending the right to life and health, of producers and
\end{abstract}


consumers, ecological agriculture involves the practice of solidarity economy, valuing small farmers and contributing to their independence, in addition to bringing important points such as sustainability, preservation of the environment, respect for local knowledge and not wasting food and resources.

Keywords: Biodynamic agriculture; Biological agriculture; Natural agriculture; Organic agriculture.

\begin{abstract}
Resumen
Cuando nos referimos a la Agroecología, nos estamos enfocando em um conjunto de principios (unidad) y, cuando nos referimos a la Agricultura Ecológica, nos referimos a las manifestaciones concretas o la materialización de esos conceptos (diversidad), a través de formas específicas de gestión. Este conjunto de iniciativas / experiências, desde uma mirada holística, que considera a complejidad e a relação entre os diversos processos que ocorrem nos agroecosistemas, configura um modelo agrícola baseado em princípios ecológicos de produção, que contrasta com a agricultura moderna. Este artigo tuvo como objetivo realizar um relevamiento bibliográfico sobre os principais sistemas de produção de base ecológica, com base no sistema orgânico. Através de descriptores previamente seleccionados, se realiza uma búsqueda ativa nas principais plataformas de investigação, artigos, discussões e tese que aborda como tema principal ou secundário as principais formas de agricultura ecológica: Biodinámica, Natural, Biológica e Orgânica. Mucho más allá de la ausencia del uso de plaguicidas, la adesión a la agricultura de base ecológica, más especificamente, la agricultura de base orgánica, es expansiva and involucra cuestiones sociales, ambientales y econômicas. Además de defender intrínsecamente el derecho a la vida y la salud, de productores y consumidores, involucra la práctica de una economia solidaria, valorando a los pequeños agricultores y contribuindo a su independencia, además de traer puntos importantes como la sostenibilidad, la preservación ambiental, el respeto por el conocimiento local y no desperdiciar alimentos y recursos.
\end{abstract}

Palabras clave: Agricultura biodinámica; Agricultura ecológica; Agricultura natural; Agricultura orgánica.

\title{
1. Introdução
}

O início da agricultura remete há mais de 10.000 anos, em que a humanidade até pouco tempo produzia alimentos de qualidade sem qualquer dependência da utilização de insumos artificialmente sintetizados. A partir do século XVI, na Europa, a adoção de práticas de manejo integrando lavoura e pecuária, a diversificação de espécies e a utilização de equipamentos de tração animal, caracterizou a Primeira Revolução Agrícola dos tempos modernos, fornecendo bases técnicas e cientificas que deram origem, no século XX, a Agricultura Biodinâmica, a Agricultura Orgânica, a Agricultura Biológica e a Agricultura Natural; agriculturas com base em princípios ecológicos (Biachini \& Medaets, 2013).

A atividade da agricultura quase sempre significa empobrecer os sistemas ecológicos naturais, do ponto de vista da biodiversidade, isto é, da quantidade de formas de vida que ali estão presentes. Quanto maior o número de espécies sejam animais ou vegetais, maior será a biodiversidade. A forma de fazer isso geralmente vai ao sentido contrário da estratégia que a natureza usa para evoluir. Assim, o grande desafio que se coloca para uma agricultura de base ecológica é o de produzir sem comprometer a preservação ou a renovação dos recursos naturais ao longo do tempo (Paulus, Muller \& Barcellos, 2000).

O século passado, em particular, foi uma época de turbulência ambiental incomum. Inúmeras práticas humanas como o desmatamento, o uso do fogo e de combustíveis de diversos tipos, a pesca, a agricultura, a industrialização, a utilização de tecnologia avançada, etc., mudaram completamente a relação do ser humano com o meio ambiente (Dias, 2015).

O conceito de qualidade do produto agrícola começa a considerar mais as questões da saúde humana. Esse conjunto de iniciativas/experiências, a partir de uma visão holística, que considera a complexidade e a relação entre os diversos processos que ocorrem nos agroecossitemas, configura um modelo de agricultura com base em princípios ecológicos de produção, que se contrapõe à agricultura moderna (Alves, Santos \& Azevedo, 2012).

Diante do exposto e tomando como fundamento a importância de conhecermos e apoiarmos formas de agricultura que promovam a sustentabilidade ambiental, o presente artigo tem como objetivo realizar uma revisão bibliográfica dos principais sistemas de agricultura de base ecológica, com ênfase na produção orgânica. 


\section{Metodologia}

Através de descritores previamente selecionados realizou-se uma busca ativa por artigos, dissertações e teses que abordavam como tema principal ou secundário as principais formas de agricultura ecológica: Biodinâmica, Natural, Biológica e Orgânica. Encontrou-se um total de 18 artigos científicos, porém três foram excluídos por não apresentarem o conteúdo do trabalho na íntegra, apenas o resumo; 4 dissertações e 1 tese. Não identificou-se a necessidade de aplicar algum critério de exclusão durante as buscas, totalizando no final 20 publicações utilizadas nesta revisão.

Foram utilizadas as plataformas de pesquisa: Scielo, Google acadêmico, Portal de periódicos da CAPES e BDTD, com a quantidade de publicações obtidas por cada, respectivamente, 10, 3, 5 e 2. Visto a relevância de considerável número de trabalhos publicados nas últimas duas décadas, não se delimitou um período de tempo para as publicações incluídas nesta revisão.

\section{Resultados e Discussão}

\section{Agricultura Biodinâmica}

O termo biodinâmico é composto por duas palavras: biológico, que se refere a uma agricultura inerente à natureza, que impulsiona os ciclos vitais através de adubação verde, consórcios e rotações de culturas; e dinâmico que está relacionado à ciência e aplicação pelo produtor dos ritmos formativos e de desenvolvimento da natureza, o que na prática agrícola acontece por meio do uso dos preparados homeopáticos à base de minerais, esterco bovino e plantas medicinais, buscando promover a vitalidade dos alimentos e manter a harmonia com os ritmos naturais e estruturação da paisagem agrícola (Leite \& Polli, 2020).

A agricultura biodinâmica possui como princípio básico entender a propriedade como um organismo individual, onde se preconizam as práticas que permitem a interação entre plantas e animais. Propõe uma visão abrangente de uma integração, considerando princípios sociais, ecológicos, culturais, técnicos e econômicos, tendo como objetivo a renovação do manejo agrícola, onde a propriedade possa viver em harmonia sem fazer uso de agrotóxicos, fertilizantes químicos e nem de maquinários pesados (Alecu \& Alecu, 2015).

A agricultura biodinâmica contribui para a criação de um ecossistema independente de recursos externos, agrotóxicos, fertilizantes químicos, entre outros. Fundamentando-se não somente no princípio de agricultura orgânica, mas também reconhecendo a "saúde" dos componentes que o integra, desde solo, plantas, animais e até mesmo o ser humano (MorrisonWhittle, Lee \& Goddard, 2017).

A biodinâmica acredita na influência que os ciclos da lua exercem nas plantas, e que seus preparados têm um poder além do cientifico, de modo que todo organismo vivo que faça parte do sistema esteja conectado ao cosmos. E assim fazem uso de calendário biodinâmico, que indicará fases astronômicas que favoreçam cada uma das atividades agrícola (Turinek, 2020).

\section{Agricultura Natural}

A concepção da agricultura natural se funda em uma perspectiva de se recuperar a energia vital original do solo, através dos respeitos as "Leis da Natureza". Esta concepção sugere que o pensamento e o sentimento do ser humano exercem grande influência no desenvolvimento das atividades produtivas. Nesta linha propõe que a atual etapa da humanidade seria de semicivilização, pois as condições materiais, não são acompanhadas daquelas espirituais, para sermos felizes (Dematte \& Filho, 2014).

Apresenta como norte a reprodução dos processos naturais próprios da natureza, evitando a ação desregrada do ser humano nos sistemas produtivos, priorizando a reciclagem energética. Suas práticas agrícolas principais giram em torno da utilização de fontes exclusivamente vegetais para a elaboração de fertilização oriunda de compostos orgânicos, além de focar 
na rotação de culturas e cobertura vegetal. Considerados como impuros, os materiais de origem animal, como o esterco bovino, não deve fazer parte da agricultura de base natural (Lopes \& Lopes, 2011).

A agricultura natural tem como premissa que o espírito é inerente aos animais, aos vegetais e todos os seres vivos, e o solo, que é o maior organismo vivo do planeta, também tem sua essência espiritual. Assim como, o alimento que é produzido segundo os princípios da Agricultura Natural além de ser saudável também tem energia vital, devido à gratidão pelo trabalho do agricultor e pela dedicação do agricultor em oferecer um alimento saudável (Gonçalves, 2016).

Enquanto método, visando promover uma produção de alimentos de elevada qualidade, livre de resíduos e essencialmente embasada em princípios de harmonia do homem com a natureza, a agricultura natural tende a favorecer o reconhecimento da multifuncionalidade da agricultura. Assim como os conceitos que fundamentam a Agricultura Natural, com seu embasamento filosófico aderem substancialmente às noções da multifuncionalidade da agricultura. (Demattê Filho, 2014).

\section{Agricultura Biológica}

Este conceito foi desenvolvido na Alemanha por Rudolf Steiner (1924) baseado na promoção da harmonia e do equilíbrio do sistema de produção (solo, plantas, animais e homem) e usa a influência do sol e da lua. De acordo com Steiner, de modo a garantir uma ligação entre todas as formas de matéria e energia no ecossistema, os elementos biológicos produzidos na exploração devem ser reincorporados como se de um corpo indivisível se tratasse (Costa et al., 2014).

Essa corrente preocupava-se com a autonomia dos produtores e com os sistemas de comercialização direta dos produtos (Caixeta \& Pedini, 2002). Leva-se em consideração o "tripé" da sustentabilidade, os aspectos ambientais, sociais e ecológicos da agricultura. Com o objetivo de melhorar a qualidade de vida dos agricultores e promover a manutenção dos agroecossistemas, os idealizadores do movimento sugerem a integração entre as unidades produtivas agrícolas e os sistemas urbanos, favorecendo a conexão entre eles e o maior aproveitamento de todos os subprodutos gerados tanto no meio rural como no urbano (Lopes \& Lopes, 2011).

Os motivos subjacentes às escolhas, comportamentos ou dietas alimentares podem ser classificados em dois grandes grupos, sendo eles: os egoísticos, ligados à saúde e bem-estar do indivíduo (como a qualidade nutritiva e a segurança alimentar e o sabor e aspeto dos alimentos consumidos) e os altruísticos, associados a preocupações éticas (com o ambiente, bem-estar animal, comércio justo e produção local) (Sautron et al., 2015).

Firmino (2006) no seu artigo A Agricultura Biológica no Algarve: um abraço entre gerações, relata que a agricultura biológica (...) é o repositório dum conhecimento milenar que chegou aos nossos dias pelo saber-fazer dos nossos antepassados, tendo sido aperfeiçoado e adaptado às exigências do mercado e às contingências ambientais e sociais que dominam no presente, beneficiando dos avanços da tecnologia para corresponder aos desideratos da sociedade moderna.

\section{Agricultura Orgânica}

Dentro das linhas da agroecologia, a agricultura orgânica é a mais propagada. Relatos encontrados em um livro intitulado "Um testamento agrícola" de 1940 aponta o inicio da agricultura orgânica no início da década de 1920. Este livro foi escrito pelo pesquisador inglês Albert Howard, após observação das práticas agrícolas de compostagem e adubação orgânica durante viagem à Índia (Filho, Ormond, Paula \& Rocha, 2002).

Nos países em que a agricultura orgânica se desenvolveu de forma considerável, percebe-se o papel imprescindível das políticas públicas e privadas, possibilitando o rápido crescimento da participação de produtos orgânicos certificados no mercado mundial. Também é importante o papel das agências de desenvolvimento nacionais e internacionais, garantindo a segurança dos alimentos, o aumento da renda dos produtores e a interrupção (ou reversão) da degradação ambiental (Borguini \& Torres, 2006; Brasil, 2007). 
A agricultura orgânica brasileira fornece predominantemente produtos de consumo direto, principalmente laticínios, conservas e hortigranjeiros, concentrando-se nos Estados de São Paulo, Minas Gerais, Espírito Santo, Paraná e Rio Grande do Sul, comumente comercializados em feiras livres e estabelecimentos de produtos naturais (Santos et al., 2012).

A principal motivação para a compra de alimentos orgânicos no Brasil parece estar ligada à saúde humana e ao meio ambiente. Existe, no entanto, um grande desconhecimento do consumidor em relação ao produto orgânico, visto que há uma grande confusão gerada pela "onda" de produtos considerados naturais, dietéticos, lights, integrais, etc. Além disso, produtos orgânicos e hidropônicos são, em geral, postos lado a lado e embalados de forma similar estrategicamente. Até mesmo alguns produtos convencionais processados e embalados em atmosfera modificada constituem outra tendência para disputar este espaço, como, por exemplo, os alimentos cortados, lavados e prontos para o consumo, já que algumas embalagens trazem dizeres como "natural, sem conservantes e aditivos" (Neto, Denuzi, Rinaldi \& Staduto, 2010).

Passados 16 anos após o lançamento da primeira portaria, em 1994, os trabalhos continuam com o objetivo de firmar a regulação do setor orgânico. Em 2007 após o alcance da regulamentação, o prazo para certificação dos produtores de orgânicos foi estendido para o final do ano de 2010, para que todos os envolvidos tivessem tempo para se adequar, pois a regulamentação não deve prejudicar os produtores, e sim, objetiva desenvolver esse setor para que ele tenha maior credibilidade e competitividade no mercado nacional e internacional (Alves, Santos \& Azevedo, 2012).

O papel da agricultura orgânica deve ser o de sustentar e aumentar a saúde do solo, das plantas, dos animais, do homem e do planeta, seja por meio do manejo do solo, do processamento dos alimentos, da distribuição ou do consumo. Entende-se que somente em solo saudável é possível produzir alimentos que vão sustentar animais e pessoas de forma saudável, influenciando a saúde das comunidades que, por sua vez, não pode ser separada da saúde do ecossistema no qual se inserem. Assim, quaisquer substâncias, sejam adubos químicos, agrotóxicos, drogas veterinárias e aditivos para o processamento dos alimentos, que possam, de alguma forma, ter efeito adverso à saúde das pessoas, dos animais, das plantas ou do ecossistema devem ser evitadas (Fonseca, 2009).

\section{Conclusão}

Por trás do aumento quantitativo da produção de insumos alimentícios, os avanços tecnológicos no setor agrícola trouxeram como consequência uma verdadeira degradação socioambiental.

Ao contrário do que possa parecer, a retomada de antigas práticas rurais livres de quaisquer tipos de aditivos químicos, mas associadas aos conhecimentos atuais sobre o "lidar" com a natureza, faz parte de uma visão de futuro onde o homem e o meio ambiente conseguem estabelecer um convívio amigável e produtivo sem a mínima intervenção possível.

Muito além da ausência do uso de agrotóxicos, a adesão a uma agricultura de base ecológica, de forma mais específica, a agricultura de base orgânica, é expansiva e envolve questões sociais, ambientais e econômicas. Além de intrinsicamente defender o direito a vida e a saúde, de produtores e consumidores, ela engaja a prática de uma economia solidária, valorizando os pequenos agricultores e contribuindo para sua independência, além de trazer consigo pontos importantes como sustentabilidade, preservação ambiental, respeito aos saberes locais e não desperdício de alimentos e recursos.

Garantir ao longo do tempo o fornecimento de alimentos de qualidade, no sentido mais amplo da palavra qualidade, é uma questão que deve ser levantada e discutida sempre que possível, no ambiente familiar, no ambiente social e no ambiente acadêmico, pois as bandeiras que levantamos hoje nos mostram o quanto estamos preocupados com o amanhã. 


\section{Referências}

Alecu, I. I., \& Alecu, E. (2015). Biodynamic agriculture versus organic farming. Journal of Biotechnology, v. 208, n. sup., p. 48-49.

Alves, A. C. O., Santos, A. L. S., \& Azevedo, R. M. M. C. (2012). Agricultura orgânica no Brasil: sua trajetória para a certificação compulsória. Revista Brasileira de Agroecologia, Porto Alegre, v.7, n.2, p.19-27.

Biachini, V., \& Medaets, J. P. P. (2013). Da Revolução verde a agroecologia: Plano Brasil agroecológico. MDA, Brasília.

Borguini, R. G., \& Torres, E. A. F. S. (2006). Alimentos orgânicos: qualidade nutritiva e segurança do alimento. Segurança Alimentar e Nutricional, v. 13, n. 2, p. 64-75.

Brasil. (2007). Ministério da Agricultura, Pecuária e Abastecimento. Decreto no 6.323, de 27 de dezembro de 2007. Regulamenta a Lei no 10.831, de 23 de Dezembro de 2003, que dispõe sobre a agricultura orgânica e dá outras providências. Diário Oficial [da] República Federativa do Brasil, Brasília, Seção 1, p. 2 .

Caixeta, I. F., \& Pedini, S. (2002). Cafeicultura orgânica: conceitos e princípios. Informe agropecuário, Belo Horizonte, v.23, n.214/215, p.15-20, jan./abr.

Costa, C. A., Correia, H. E., Correia, P., Costa, D., Gaião, D., Guiné, R., Coelho, C., Costa, J. M., Monteiro, A., Oliveira, J., Pinto, A., Rodrigues, P., Castro, M., Guerra, L. T., Seeds, C., Coli, C., Macdonald, J., Radics, L., Soylu, S., Arlans, M., Tóthová, M., Tóth, P., \& Basile, S. (2016). Organic Farming e-book. Estrategia y Organización S. A., n. 5, oficina 4.

Demattê Filho, L. C. (2014). Sistema agroalimentar da avicultura fundada em princípios da agricultura natural: multifuncionalidade, desenvolvimento territorial e sustentabilidade. Tese (Doutorado em Ecologia Aplicada) - Universidade de São Paulo, São Paulo, $251 \mathrm{f}$.

Dias, R. (2015). Sustentabilidade: origem e fundamentos: educação e governança global: modelo de desenvolvimento. Atlas, São Paulo.

Filho, P. V., Ormond, J. G. P., Paula, S. R. L., Rocha, L. T. (2002). Agricultura Orgânica: Quando o passado é futuro. BNDES Setorial, Rio de Janeiro, n. 15, p. 3-34.

Firmino, A. (2006). A agricultura Biológica no Algarve: um abraço entre gerações. In: Desenvolvimento e território - espaços rurais pós-agrícolas e novos lugares de turismo e lazer. Centro de Estudos Geográficos - Universidade de Lisboa, 362p.

Fonseca, M. F. A. C. (2009). Agricultura orgânica: regulamentos técnicos e acesso aos mercados dos produtos orgânicos no Brasil. PESAGRO-RIO. 119 p.

Gonçalves, M. O. (2016). A agricultura natural como referência para o desenvolvimento sustentável: Centro de Pesquisa Mokiti Okada. Dissertação (Mestrado em Políticas Públicas) - Universidade Estadual de Maringá, Maringá, 82p.

Leite, A. B., \& Polli, H. Q. (2020). Agricultura Orgânica no Brasil com enfoque na Agricultura Biodinâmica. Interface Tecnológica, v.17, n. 1, 2020.

Lopes, P. R., \& Lopes, K. C. S. A. (2011). Sistemas de produção de base ecológica - a busca por um desenvolvimento rural sustentável. Revista Espaço de Diálogo e Desconexão, v.4, n. 1 .

Morrison-Whittle, P., Lee, S. A., \& Goddard, M. R. (2017). Fungal communities are differentially affected by conventional and biodynamic agricultural management approaches in vineyard ecosystems. Agriculture, Ecosystems \& Environment, v. 246, p. 306-313.

Neto, N. C., Denuzi, V. S. S., Rinaldi, R. N., \& Staduto, J. A. R. (2010). Produção orgânica: uma potencialidade estratégica para a agricultura familiar. Revista Percurso. Maringá, v. 2, n. 2, p. 73-95.

Santos, J. O., Santos, R. M. S., Borges, M. G. B., Ferreira, R. T. F. V., Salgado, A. B., \& Santos Segundo, O. A. (2012). A evolução da agricultura orgânica. Revista Brasileira de Gestão Ambiental, Pombal, v.6, n.1, p.35-41.

Sautron, V., Péneau, S., Camilleri, G. M.; Muller, L.; Ruffieux, B., Herceberg, S., \& Méjean, C. (2015). Validity of a questionnaire measuring motives for choosing foods including sustainable concerns. Appetite. v. 87, p.90-97.

Turinek, M. (2020). Biodynamic soil fertility management in fruit crops. In: Srivastava, A. K.; HU, C. - Fruit Crops: Diagnosis and Management of Nutrient Constraints. Elsevier, p. 393-400. 